\title{
ESTANDARIZACIÓN DE UNA BATERÍA PARA LA EVALUACIÓN DE FACTORES DE RIESGO PSICOSOCIALES LABORALES EN TRABAJADORES COLOMBIANOS
}

\author{
Viviola Gómez ${ }^{1 *}$, Sebastián Segura Camacho ${ }^{2}$, Diego Castrillón ${ }^{2}$, Lyria Esperanza Perilla ${ }^{1}$ \\ ${ }^{1}$ Grupo de Investigación Estrés y Salud, Departamento de Psicología, Universidad de los Andes, Colombia, \\ ${ }^{2}$ Empresa de Consultoría Prax S.A.S., Colombia.
}

\author{
Recibido, mayo 13/2015 \\ Concepto evaluación, mayo 19/2016 \\ Aceptado, mayo 25/2016
}

\begin{abstract}
Referencia: Gómez, V., Segura Camacho, S., Castrillón, D. \& Perilla, L. E. (2016). Estandarización de una batería para la evaluación de factores de riesgo psicosociales laborales en trabajadores colombianos. Acta Colombiana de Psicología, 19(2), 221-238. DOI: 10.14718/ACP.2016.19.2.10
\end{abstract}

Resumen

En 2010 se desarrolló una batería de instrumentos para evaluar factores psicosociales laborales de riesgo para la salud, en respuesta a la Resolución 2646 de 2008 del Ministerio de la Protección Social de Colombia. Sin embargo, esta cuenta con algunas limitaciones que, a partir de la construcción y validación de una nueva batería, en el presente estudio se buscan superar. La nueva batería ofrece recursos adicionales para la evaluación de estos factores: incorpora los instrumentos e indicadores centrales de los modelos demanda-control-apoyo social y desequilibrio esfuerzo-recompensa, y los factores intralaborales no contemplados en dichos modelos, pero que la Resolución considera necesarios, se midieron con pruebas preexistentes o desarrolladas por los autores. Con los datos recolectados es posible calcular indicadores globales de demanda, control y apoyo social; además de condiciones familiares y sociales de riesgo, afrontamiento, personalidad e indicadores de salud y bienestar. Para la validación, la batería se aplicó a una muestra de 16.095 trabajadores de diferentes ocupaciones y municipios colombianos. Los análisis de consistencia interna y validez permiten afirmar que la batería es sencilla de aplicar en papel o por computador, permitirá comparar ocupaciones, obtener puntuaciones unificadas por variable, ofrecer un diagnóstico de un número importante de las variables sugeridas en la Resolución y comparar los resultados de los trabajadores colombianos con los de otros países.

Palabras clave: factores laborales de riesgo psicosocial, Resolución 2646 de 2008, modelo demanda-control-apoyo social, modelo desequilibrio esfuerzo-recompensa, estrés laboral, evaluación.

\section{STANDARDIZATION OF A BATTERY OF TESTS TO ASSESS PSYCHOSOCIAL RISK FACTORS AT THE WORKPLACE AMONG COLOMBLAN WORKERS}

\begin{abstract}
A battery of questionnaires to assess psychosocial risk factors at work was developed in 2010 in response to Resolution 2646 created by the Colombian Ministry of Social Protection. However, this battery presents some theoretical and practical limitations. A new battery of instruments has been designed and validated that includes instruments and risk indicators of the demand-control-social support and the effort-reward imbalance models. Other factors, not included in these models, but that Resolution 2646 suggests should be assessed, have also been added, and with this additional information, the new battery allows us to also calculate a "global indicator" of demand, control, and social support; family and social risk conditions, coping and personality; and health and wellbeing. The new battery was administered to a sample of 16,095 workers from different occupations and representative Colombian regions. An analysis of the various domains indicates that internal consistency of the various scales is high. The new battery has the following properties: it is simple to use in paper format or when administered by computer, it enables comparison between occupations, it offers unified scores for each variable, and provides information to assess the risk factors suggested by Resolution 2646. In addition, it will make it possible to compare the results obtained when analyzing Colombian workers with those obtained from studies of workers from other countries.

Keywords: psychosocial risk factors, work, Resolution 2646 of 2008, demand-control-social support model, effort-reward imbalance model, job stress, survey.
\end{abstract}

\footnotetext{
*Viviola Gómez. Teléfono 57-1-3324365 ext.2546. vgomez@uniandes.edu.co. Agradecemos a las empresas, trabajadores y personas que hicieron posible este trabajo; a Henrique G. Abreu y Alexander Steshenko, quienes cuidadosamente diseñaron y prepararon los sistemas de información y datos requeridos para su desarrollo; y, finalmente, a los doctores Robert Karasek y Johannes Siegrist por autorizarnos el uso de las pruebas JCQ y ERI.
} 


\title{
PADRONIZAÇÃO DE UMA BATERIA PARA A AVALIAÇÃO DE FATORES DE RISCO PSICOSSOCIAIS TRABALHISTAS EM TRABALHADORES COLOMBIANOS
}

\author{
Resumo
}

\begin{abstract}
Em 2010, desenvolveu-se uma bateria de instrumentos para avaliar fatores psicossociais trabalhistas de risco para a saúde, em resposta à Resolução 2646 do Ministério da Proteção Social da Colômbia. Contudo, esta conta com algumas limitações que, a partir da construção e da validação de uma nova bateria, neste estudo se pretendem superar. Além disso, a nova bateria oferece recursos adicionais para a avaliação desses fatores: a presente bateria incorpora os instrumentos e os indicadores centrais dos modelos demanda-controle-apoio social e desiquilíbrio esforço-recompensa e os fatores internos do trabalho não considerados nesses modelos, mas que a Resolução considera necessários, mediram-se com testes preexistentes ou desenvolvidos pelos autores. Com os dados coletados, é possível calcular indicadores globais de demanda, controle e apoio social; além de condições familiares e sociais de risco, enfrentamento, personalidade e indicadores de saúde e bem-estar. Para a validação, a bateria foi aplicada a uma amostra de 16095 trabalhadores de diferentes cargos e municípios colombianos. As análises de consistência interna e validade permitem afirmar que a bateria é simples de aplicar em papel ou digital, permitirá comparar cargos, obter pontuações unificadas por variável, oferecer um diagnóstico de um número importante das variáveis sugeridas na Resolução bem como permitirá comparar os resultados dos trabalhadores colombianos com os de outros países.

Palavras-chave: fatores trabalhistas de risco psicossocial, Resolução 2646 de 2008, modelo demanda-controle-apoio social, modelo desiquilíbrio esforço-recompensa, estresse profissional, avaliação.
\end{abstract}

En 2008 el Ministerio de la Protección Social de Colombia publicó la Resolución 2646 mediante la cual reguló las responsabilidades de los empleadores colombianos frente a la prevención, diagnóstico, intervención y control de los factores de riesgo psicosociales laborales (Ministerio de la Protección Social, 2008). Y en 2010, dado que en dicha Resolución se hizo énfasis en la importancia de usar instrumentos validados en Colombia para medir estas variables, el Ministerio contrató a la Pontificia Universidad Javeriana para que diseñara una batería de instrumentos que cumpliera con este objetivo (Ministerio de la Protección Social, 2010).

La batería del Ministerio ofrece beneficios, como contar con algunos cuestionarios de uso y dominio público; incluir gran parte de las variables e información que el Ministerio espera sean tomadas en cuenta; y ofrecer, además de los cuestionarios, otros recursos para la evaluación del riesgo psicosocial laboral. Sin embargo, se han identificado algunas limitaciones prácticas y teóricas en el cuestionario, el más usado de todos los recursos de evaluación de la batería. Estas limitaciones son: la restricción para ser aplicada por computador (Ministerio de la Protección Social, 2011); la imposibilidad de comparar ocupaciones, ya que solo evalúa tipos de cargo (jefatura, profesionales, técnicos, auxiliares-operarios); la alta complejidad para la planeación y aplicación ${ }^{1}$; la imposibilidad de obtener una puntuación

\footnotetext{
La batería cuenta con dos formas, de acuerdo con el tipo de cargo que desempeñe el trabajador; hecho que obliga a que antes de iniciar la evaluación se deba invertir tiempo y esfuerzo clasificando a los trabajadores para determinar el tipo de forma apropiado. Y en caso de aplicar la prueba incorrecta, sería necesario anularla o evaluar nuevamente.
}

unificada por variable ${ }^{2}$; un diagnóstico incompleto, ya que no cubre todas las variables señaladas en la Resolución; $\mathrm{y}$, finalmente, para efectos de investigación y estudios epidemiológicos, el hecho de que la batería emplee cuestionarios nuevos, que no se han utilizado antes y que son diferentes en términos conceptuales a los propuestos por otros autores, impide comparar los resultados obtenidos con los de grupos evaluados previamente en Colombia $\mathrm{u}$ otras partes del mundo.

Asimismo, la batería cuenta con limitaciones de tipo teórico, pues los autores afirman que para su desarrollo se basaron en el modelo de Desequilibrio Esfuerzo-Recompensa (DER), el de Demanda-Control-Apoyo Social (DCA), en la estructura factorial del Copenhagen Psychosocial Questionnaire y la teoría del estrés de Ivancevich y Matteson; además de apoyarse, en las necesidades del país y las investigaciones y experiencias previas de los autores (Villalobos, Vargas, Rondón y Felknor, 2013). Sin embargo, de las contribuciones teóricas de los modelos DCA y DER, utilizaron algunos de sus componentes, como demanda psicológica, control laboral, recompensas y apoyo social, pero omitieron otros que forman parte integral de los mo-

\footnotetext{
2 Las dos formas de la batería tienen baremos diferentes, lo que obliga a que el cálculo de una variable se realice dos veces, uno para los tipos de cargos evaluados con la forma A y otra para los cargos evaluados con la forma B. Por lo anterior, cada diagnóstico empresarial se convierte en un proceso muy largo, de difícil comprensión en las organizaciones, que restringe las posibilidades de análisis consolidados y que refuerza la idea en las empresas de que la intervención se hace sobre los trabajadores y no sobre las causas del factor de riesgo.
} 
delos, como esfuerzo, sobrecompromiso, tensión laboral y desequilibrio esfuerzo-recompensa.

Estos componentes omitidos, principalmente los relacionados con la interacción entre demanda y control, y entre esfuerzos y recompensas, representan lo que, de acuerdo con los modelos, son los factores psicosociales laborales de mayor riesgo para la salud. De esta forma, solo se puede evaluar el impacto de la demanda y el control, pero no su relación, y no es posible calcular el desequilibrio entre esfuerzos y recompensas.

En otras palabras, dado que la batería no incluyó todos los factores laborales ni los indicadores relacionales de los cuales se derivan las principales predicciones teóricas de los modelos, se evidencia que el uso de estos se hizo de forma incompleta.

La incoherencia teórica señalada es de gran importancia ya que tal como están descritos y medidos los factores intralaborales en la batería del Ministerio, pareciera que todos ellos tienen la misma capacidad de afectar la salud de los trabajadores, lo cual no es coherente con las predicciones de los modelos de Karasek (1979) y Siegrist (2002) ni con otros datos empíricos que los sustentan (ver citas más adelante) que sostienen que no todas las variables intralaborales, extralaborales o personales son igualmente relevantes en términos del riesgo que representan para la salud; que algunos factores psicosociales laborales, como demanda laboral, control laboral, esfuerzos invertidos o recompensas a los esfuerzos invertidos en el trabajo, son más relevantes que otros; y que el riesgo para la salud se puede predecir de manera mucho más precisa cuando se toma en cuenta la interacción entre estos factores, y no solo la información de cada uno de ellos de manera independiente.

Teniendo esto en cuenta, el objetivo del presente trabajo fue construir y validar una batería que superara algunas de las limitaciones encontradas en la batería de factores de riesgo psicosocial del Ministerio de la Protección Social y que, en concreto, cumpliera con las siguientes características: a) facilite la evaluación de trabajadores tanto por computador como en papel; b) reduzca y simplifique los esfuerzos logísticos de evaluación; c) permita evaluar una mayor cantidad de las variables sugeridas por la Resolución 2646, pero al mismo tiempo ofrezca la posibilidad de calcular indicadores que, según la literatura, representan el mayor riesgo para la salud de los trabajadores; d) posibilite comparar los datos de trabajadores colombianos con datos de estudios previos (nacionales o internacionales), y e) que facilite la toma de decisiones para la prevención e intervención, sustentadas teórica y empíricamente.

Cabe agregar que disponer de más de un instrumento validado en Colombia aporta mayores opciones para la medición, que pueden suplir las necesidades de la gran diversidad de empresas, actividades económicas y realidades e intereses de evaluación que existen en el país.

\section{Modelos teóricos}

El Ministerio de la Protección Social de Colombia, con el objetivo de establecer las condiciones y variables por evaluar con la batería, definió en la Resolución 2646 de 2008 los factores psicosociales como "los aspectos intralaborales, los extralaborales o externos a la organización y las condiciones individuales o características intrínsecas del trabajador, los cuales en una interrelación dinámica, mediante percepciones y experiencias, influyen en la salud y el desempeño de las personas" (Ministerio de la Protección Social, 2008, p. 3). Esta definición guió las variables y condiciones que se sugieren evaluar en la Resolución 2646.

La batería que se presenta en este trabajo se basa en la definición de la OIT/OMS (Comité Mixto OIT-OMS, 1984), que coincide parcialmente con la del Ministerio, pero hace énfasis en los factores psicosociales laborales, consisten en interacciones entre el trabajo, su medioambiente, la satisfacción en el trabajo y las condiciones de su organización, por una parte, y por la otra, entre las capacidades del trabajador, sus necesidades, su cultura y su situación personal fuera del trabajo, todo lo cual, a través de percepciones y experiencias, puede influir en la salud, el rendimiento y la satisfacción en el trabajo.

Esta definición resalta que los efectos de dichos factores pueden ser tanto positivos como negativos, y dependen del ajuste entre las capacidades y características del empleado con las demandas y condiciones del ambiente de trabajo. Y adicionalmente, hace hincapié en la percepción y experiencia de estos factores, y en cómo pueden desembocar en daños en la salud de los individuos y de la organización.

Esta conceptualización permite que, a la hora de evaluar los factores de riesgo psicosocial en el contexto laboral, se tomen decisiones sustentadas en teorías generales del estrés, y específicamente en el estrés de origen laboral, para así, teniendo en cuenta que las condiciones psicosociales en el trabajo pueden convertirse en factores de riesgo o de protección, determinar las variables de mayor interés.

Específicamente, la relación entre los factores psicosociales de origen laboral, el estrés y la salud se sustenta en varios modelos teóricos, dentro de los cuales se pueden destacar el DCA (Demanda - Control - Apoyo Social), desarrollado por Karasek (1979; Karasek y Theorell, 1990) y el DER (Desequilibrio Esfuerzo - Recompensa), por Siegrist (2002). 
Modelo de demanda, control y apoyo social (DCA)

Este modelo se enfoca esencialmente en el contenido del trabajo; su hipótesis principal es que los trabajos que probablemente más malestar y enfermedad generan son aquellos en los que las personas se enfrentan a la combinación simultánea de altas demandas y bajo control (Tensión Laboral o Job Strain); sus componentes principales son las demandas psicológicas de la labor y la posibilidad que se tenga, durante el trabajo, de usar (o aprender) habilidades y tomar decisiones en torno a la forma de desarrollar las tareas (control laboral o margen de decisión) (Karasek y Theorell, 1990).

Por otro lado, este modelo incluye al apoyo social en el lugar de trabajo, que se refiere a los niveles globales de interacción social (con supervisores o compañeros) que ofrecen apoyo en el trabajo y que podrían afectar el bienestar de los trabajadores, actuando como un amortiguador (moderador) entre los estresores psicosociales laborales y los resultados adversos en la salud (Karasek, Gardell y Lindell, 1987).

En este sentido, la peor combinación que podría haber: altas demandas laborales, baja libertad de decisión y bajo apoyo social, tendría las consecuencias más adversas para la salud (Johnson, Hall y Theorell, 1989; Kinman, Jones y Kinman, 2006; Winefield, Gillespie, Stough, Dua, Hapuarachchi y Boyd, 2003). La inseguridad laboral, a pesar de no estar contemplada en las predicciones más importantes del modelo, es tomada en cuenta porque diversos estudios señalan sistemáticamente que es un indicador independiente, y muy potente, de problemas de salud (Sverke y Hellgren, 2002; Sverke, Hellgren y Näswall, 2002).

El impacto en la salud por parte de las condiciones estresantes de trabajo señaladas por este modelo ha sido investigado por muchos años; específicamente, un gran número de estudios en diversos países han demostrado el efecto del trabajo con tensión laboral (combinación de altas demandas y bajo control) sobre la presión sanguínea alta, la enfermedad coronaria, el malestar psicológico y desórdenes musculoesqueléticos, entre otros (Ganster y Schaubroek, 1991; Johnson y Hall, 1988; Johnson, Stewart, Hall, Fredlund y Theorell, 1996; Karasek, Baker, Marxer, Ahlbom y Theorell, 1981; Kristensen, 1995, 1996; Schnall, Belkic, Landsbergis y Baker, 2000; Theorell y Karasek, 1996, Van der Doef y Maes, 1999).

\section{Modelo de desequilibrio entre esfuerzo y recompensa (DER)}

El modelo de Siegrist (2002), denominado effort-reward imbalance o desequilibrio esfuerzo-recompensa (DER) se enfoca en la interacción entre persona y ambiente en el contexto de las organizaciones sociales; y se centra en la noción de reciprocidad social, un principio fundamental de la conducta interpersonal adquirido evolutivamente, caracterizado por los esfuerzos mutuos de cooperación que se hacen sobre la expectativa de que los esfuerzos serán igualados por las respectivas recompensas. En este sentido, cuando falla la reciprocidad se amenaza el principio fundamental, lo cual genera fuertes emociones negativas y respuestas de estrés sostenidas; mientras que cuando las recompensas sociales por los esfuerzos realizados son apropiadas, las emociones y reacciones del organismo promueven la salud, el bienestar y la sobrevivencia (Siegrist, 2002).

Este modelo afirma que las experiencias estresantes en el trabajo y sus consecuentes efectos negativos en la salud, resultan de la percepción recurrente de desequilibrio entre mucho esfuerzo o demandas externas (esfuerzo extrínseco) y bajas recompensas (dinero, estima o control del estatus laboral); en otras palabras, una falta de reciprocidad entre costos y beneficios. Las ganancias o beneficios (recompensas), de acuerdo con el modelo DER, se distribuyen a las personas que trabajan, a través de tres sistemas: dinero, estima y control del estatus laboral; es decir, control de las posibilidades de promoción y de seguridad laboral. El modelo, además, afirma que la combinación de este desequilibrio con la característica personal de excesivo nivel de compromiso con el trabajo (esfuerzo intrínseco o sobrecompromiso) aumenta la tendencia a una activación autonómica elevada y los problemas de salud que se le asocian.

Se han realizado algunos estudios que han establecido una relación entre el DER e indicadores de salud como el riesgo de enfermedad coronaria (Bosma, Peter, Siegrist y Marmot, 1998), agotamiento mental (Calnan, Wainwright y Almond, 2000) y la depresión (Tsutsumi, Kayaba, Theorell y Siegrist, 2001).

\section{Uso conjunto de los modelos DCA y DER}

Los modelos DCA y DER cuentan con características de gran relevancia en el contexto del presente trabajo tales como: (a) son modelos analíticos que seleccionan múltiples factores psicosociales intralaborales que pueden ser fuentes de estrés laboral con base en supuestos teóricos y resultados empíricos derivados del estudio científico del estrés; (b) Los factores psicosociales señalados por los modelos afectan a todas las ocupaciones y no solamente a algunas de ellas; $\mathrm{y}$ (c) muchos autores abogan por el uso conjunto de los dos modelos (Calnan et al., 2000; Peter, Siegrist, Hallqvist, Reuterwall y Theorell, 2002). Dada la diferencia en énfasis entre control y recompensas, las implicaciones para las políticas de cambio son distintas.

Debido a las ventajas que ofrece el uso de instrumentos derivados de modelos teóricos de comprobada utilidad teórica y práctica, cuya capacidad de predicción ha sido evaluada ampliamente y de los cuales existen datos publicados de 
trabajadores del mundo entero, para la construcción de esta batería se consideró apropiado incorporar los instrumentos derivados de estos modelos (Job Content Questionnaire [JCQ] y Effort Reward Imbalance [ERI] con el fin de evaluar los factores psicosociales intralaborales. Dichos instrumentos, además de haber mostrado propiedades psicométricas adecuadas en estudios previos en Colombia (Gómez, 2010; Gómez, 2011), permitirán comparaciones de los trabajadores colombianos con los de otros países y le facilitarán a los empleadores priorizar factores para la intervención en caso de que varios de ellos señalen algún nivel de riesgo para la salud de sus trabajadores.

Como los instrumentos derivados de los modelos DCA y DER no cubren todas las variables intralaborales que la Resolución 2646 invita a evaluar, la batería objeto de este estudio incluyó otros instrumentos existentes y previamente validados en Colombia o en otros países. También se desarrollaron y validaron instrumentos adicionales, cuando esto fue necesario. Los factores intralaborales que la resolución incluye pueden considerarse conceptualmente como ejemplos de demandas laborales, formas de control o de apoyo social en el trabajo. A partir de los datos de esta batería es posible calcular los indicadores de demanda, control y apoyo social derivados del JCQ; indicadores de esfuerzo, recompensa y sobrecompromiso derivados del DER; el indicador de desequilibrio DER y el indicador de tensión laboral original del JCQ; y finalmente, es posible calcular unos indicadores globales de demanda, control y apoyo social que combinan la información del JCQ y del ERI con la de las demás variables intralaborales descritas en la resolución 2646.

Como se ha visto, existe una extensa bibliografía sobre los factores psicosociales en el trabajo y sus consecuencias sobre el individuo; sin embargo, los estresores psicosociales también pueden provenir de fuentes distintas al trabajo (como la familia o condiciones sociales o personales). En este sentido, la literatura sobre el estrés y las diferencias individuales que moderan sus efectos, y particularmente, en el estrés de origen laboral, es abundante (Lazarus, 1999; Lundberg y Cooper, 2011; Semmer y Meier, 2009).
Teniendo en cuenta lo anterior, cuando se tiene interés por conocer, prevenir y controlar las condiciones que pueden afectar la salud de los trabajadores, la conceptualización realizada señala que, además de evaluar las condiciones psicosociales intralaborales de mayor riesgo para la salud, es necesario tener en cuenta las condiciones familiares o sociales que pudieran interactuar con el trabajo; las características de personalidad que representen un riesgo al interactuar con las condiciones laborales de riesgo; las formas de afrontamiento de los estresores, especialmente de origen laboral; y algunos indicadores de condiciones de salud y enfermedad, como la percepción de salud, reacciones psicosomáticas al estrés, vitalidad y satisfacción laboral.

En coherencia con esta conceptualización, además de los instrumentos para evaluar factores psicosociales intralaborales, en la batería se incluyeron instrumentos que permiten la valoración de tendencias de afrontamiento y características de personalidad que pueden incrementar o disminuir la vulnerabilidad al estrés y sus efectos. Finalmente, se incluyeron pruebas para evaluar algunos indicadores de condiciones de salud y enfermedad tales como la percepción de salud, las reacciones psicosomáticas ante el estrés, la vitalidad y la satisfacción laboral.

\section{MÉTODO}

\section{Tipo de estudio}

Se realizó un estudio transversal, usando instrumentos de autorreporte.

\section{Participantes}

Con base en el universo de trabajadores colombianos afiliados al Sistema de Riesgos Laborales (véase Tabla 1), se calculó que la muestra mínima requerida para que dicho universo estuviera representado de forma adecuada debía ser de 1539 trabajadores, distribuidos por sector económico y región, tal como se describe en la Tabla 2.

Tabla 1.

Universo de trabajadores afiliados al sistema de seguridad social colombiano

\begin{tabular}{|c|c|c|c|c|c|c|}
\hline Región & Sector & Servicios & Comercio & Industria & Agrícola & Total \\
\hline Andina & & 2741753 & 723799 & 1524510 & 241218 & 241218 \\
\hline Caribe & & 532769 & 111416 & 244278 & 29821 & 918284 \\
\hline Pacífica & & 6818 & 1831 & 2514 & 43 & 11206 \\
\hline Orinoquía & & 27100 & 4532 & 28896 & 2733 & 63261 \\
\hline Amazonía & & 93285 & 18556 & 42144 & 15398 & 169383 \\
\hline Total & & 3401725 & 860134 & 1842342 & 289213 & 6393414 \\
\hline
\end{tabular}

Nota: Datos con corte al primero de abril de 2013. Fuente: RLDatos de la Federación de Aseguradores Colombianos (FASECOLDA). 
Tabla 2.

Distribución de la muestra mínima necesaria por sector económico y región del país

\begin{tabular}{|c|c|c|c|c|c|c|}
\hline Región & Sector & Servicios & Comercio & Industria & Agrícola & Total \\
\hline Andina & & 658 & 166 & 356 & 56 & 1236 \\
\hline Caribe & & 95 & 24 & 51 & 8 & 178 \\
\hline Pacífica & & 47 & 12 & 25 & 4 & 88 \\
\hline Orinoquía & & 2 & 1 & 2 & 1 & 6 \\
\hline Amazonía & & 17 & 4 & 9 & 1 & 31 \\
\hline Total & & 819 & 207 & 443 & 70 & 1539 \\
\hline
\end{tabular}

Nota: Muestra con un error tipo 1 de 0,05 , una estimación de la frecuencia del $50 \%$ y una variación de la frecuencia del $5 \%$ con espera de dos colas.

Para la selección de participantes se invitó a participar aproximadamente a 1000 empresas afiliadas a una Administradora de Riesgos Laborales. De estas, 106 aceptaron participar, y 16.095 de sus trabajadores respondieron completamente la batería. Este muestreo no se realizó al azar, sino que se hizo por conveniencia. Sin embargo, dado el tamaño y la distribución de la muestra no hay razones para pensar que los trabajadores participantes presenten algún tipo de sesgo relacionado con sus condiciones psicosociales de trabajo.
En la tabla 3 se muestran las características sociodemográficas de la muestra. Es importante señalar que las cuotas se cumplieron ampliamente, excepto en los sectores agrícola e industrial que solo pudieron ser completamente representados en la región andina. La edad promedio de los participantes fue de 34.8 años (DS=9.2); y el $61.5 \%$ de la muestra era de sexo femenino mientras que el $38.5 \%$, masculino. Datos detallados de escolaridad y de cargo pueden apreciarse en la Tabla 3.

Tabla 3.

Descripción de la muestra final

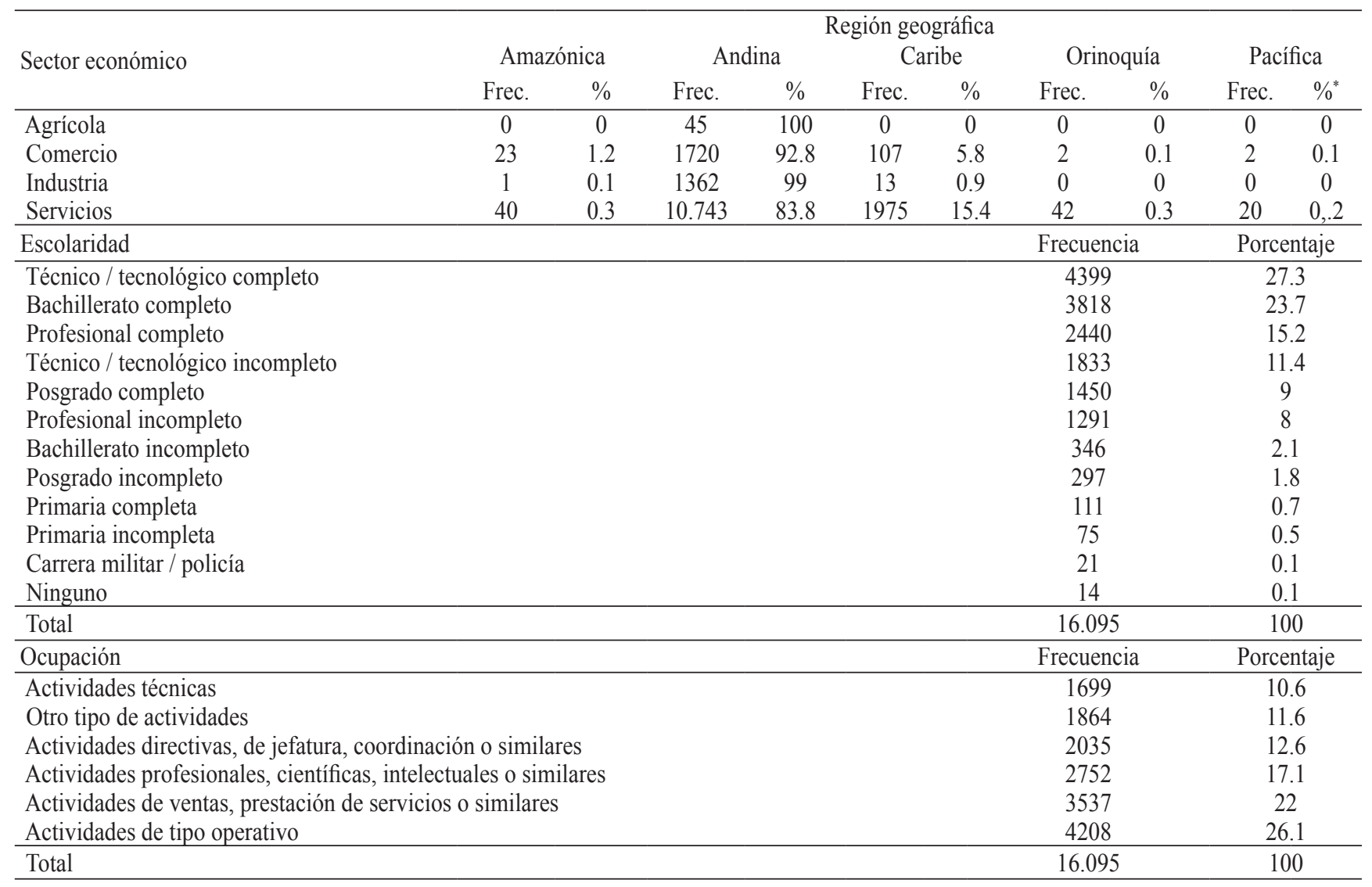

Nota: : Los porcentajes (\%) corresponden a la fila, es decir, por sector económico. 


\section{Instrumentos}

La selección de los instrumentos que formaron parte de la batería se definió de acuerdo con los siguientes criterios: a) que no tuvieran limitaciones para ser aplicados en papel o a través de medios electrónicos; b) que la planeación, aplicación y calificación de la prueba fuera sencilla; c) que fueran válidos y confiables; d) que tuvieran un modelo teórico de respaldo cuya validez, relevancia o sustento hubiera sido publicado en revistas nacionales o internacionales; y e) que, de ser posible, hubieran sido validados con muestras colombianas.

La batería se compuso de los siguientes cuestionarios y escalas, los cuales cumplieron con los criterios definidos:

\section{Cuestionario del contenido del trabajo (Job Content Questionnaire-JCQ)}

Instrumento diseñado para medir características psicosociales de las condiciones en que se realiza el trabajo, de acuerdo con el modelo DCA(Johnson y Hall, 1988; Karasek, 1979; Karasek y Theorell, 1990). Los resultados de estudios de validación en Japón, Canadá y Bélgica, entre otros, muestran, en general adecuada confiabilidad de las escalas (alfa de Cronbach entre 0.6 y 0.8 ), una estructura factorial similar al cuestionario original, además de una adecuada validez predictiva con diferentes indicadores de salud (cardiovascular, satisfacción laboral, ansiedad, depresión, afectividad negativa) (Brisson et al., 1998; Kawakami y Fujugaki, 1996; Kawakami, Kobayahi, Araki, Haratani y Furui, 1995; Pelfrene et al., 2001; Sale y Kerr, 2002). En Latinoamérica, Araújo y Karasek (2008), Cedillo y Karasek (2003) y Gómez (2011) han publicado resultados de trabajos similares.

\section{Cuestionario de desequilibrio esfuerzo-recompensa (DER)}

Instrumento diseñado para evaluar los componentes del Modelo DER (Siegrist, 2002) que se ha validado y evaluado psicométricamente, con excelentes resultados, en varios países e idiomas, como por ejemplo en Corea (Eum et al., 2007), Brasil (Griep et al., 2009), Grecia (Msaouel et al., 2012), y Francia (Niedhammer, Siegrist, Landre, Goldberg y Leclerc, 2000). Específicamente, en una versión en español fue evaluada por Macías et al. (2003), se informó que la consistencia interna fue satisfactoria para la recompensa y esfuerzo intrínseco, y se observaron valores alfa de Cronbach superiores a 0,80. Esta misma escala en español fue evaluada en Colombia por Gómez (2010), concluyendo que el cuestionario, utilizado con las ocupacionales colombianas, presenta características psicométricas similares a las del instrumento original y las traducciones validadas en otros idiomas.

\section{Cuestionario factores de riesgos psicosociales}

El cuestionario Factores de Riesgo Psicosociales de Bocanument-Norby (1993) es un instrumento que mide diversos factores intralaborales y posibles alteraciones físicas y psicológicas derivadas del trabajo. Lastimosamente, de acuerdo con Sarsosa, Arenas y Charria (2011), en ninguna de las publicaciones que reportan haber usado el instrumento (Bahamón y Zuluaga, 1993; Bocanument, 1994; Bocanument y Berjan, 1996) hay información disponible sobre las propiedades psicométricas de la prueba. De este cuestionario solo se utilizó la escala que evalúa manifestaciones del estrés tales como síntomas fisiológicos, cognitivos, emocionales y comportamentales. Aunque la prueba no cumple todos los criterios utilizados en este estudio para la selección de instrumentos, se decidió utilizarla debido a la facilidad de acceso y al amplio uso que tiene en Colombia. Con su inclusión se espera ofrecer a la comunidad académica información sobre la consistencia interna de la escala seleccionada.

\section{Cuestionario de Salud SF-36}

Esta prueba fue diseñada por John E. Ware Jr., como parte del estudio Medición de la Calidad de Vida (Measures of Quality of Life Core Survey, MOS) desarrollado por la corporación RAND. De las 40 escalas evaluadas en el estudio se seleccionaron 8 para conformar el SF-36, por ser las más usadas entre los cuestionarios de salud y las más sensibles de variar por enfermedad y tratamientos (Ware, 2013). La utilidad del cuestionario para detectar morbilidad y población enferma se ha documentado en múltiples estudios que han evaluado problemas de salud tales como artritis, dolor de espalda, cáncer, problemas cardiovasculares, enfermedad obstructiva crónica y depresión (Turner-Bowker, Bartley, y Ware, 2002). Además, se han realizado más de 500 investigaciones que han involucrado investigadores de 22 países (Ware, 2013).

En Colombia se han realizado varios estudios con la prueba, entre los que se encuentra el de Lugo, García y Gómez (2006), que concluye que esta es confiable para evaluar calidad de vida. Puntualmente, en esta batería se utilizaron las escalas de Salud General, Salud Mental y Vitalidad, tal como lo han hecho otros instrumentos de amplia aceptación como el ISTAS 21 (Moncada, Llorens y Kristensen, 2004).

\section{Escala de satisfacción laboral de Whitehall II}

Esta escala evalúa el nivel de satisfacción de un empleado con su trabajo. La prueba original fue construida por Marmot en 1967 en el marco del Whitehall Study II (Marmot, 2013). Posteriormente fue incorporada en el Copenhagen 
Psychosocial Questionnaire(COPSOQ), el cual, incluyendo la variable de satisfacción, ha sido utilizado, adaptado y baremado en varios países (Alvarado, Marchetti, Villalón, Hirmas y Pastorino, 2009; Blanch, Sahagún y Cervantes, 2010; Nübling, Stößel, Hasselhorn, Michaelis y Hofmann, 2006). En la última versión del COPSOQ se encontró un alfa de Cronbach de 0.82 para la escala de satisfacción (Pejtersen, Kristensen, Borg y Bjorner, 2010).

La adaptación al español de la escala fue realizada por el Instituto Sindical de Trabajo, Ambiente y Salud de España en el marco de la adaptación del COPSOQ Danés a España (Moncada et al., 2004) arrojando resultados consistentes con los daneses y alemanes. Aunque la escala de satisfacción ha sido estandarizada en español y se ha usado en varias investigaciones colombianas, no se cuenta con suficiente evidencia sobre su confiabilidad en este contexto, por lo cual el uso de esta escala no solo será de utilidad para este estudio, sino que también proporcionará información sobre sus propiedades psicométricas.

\section{Inventario de personalidad de Eysenck, EPI}

El objetivo de esta prueba es valorar los rasgos de personalidad como psicoticismo, extraversión, neuroticismo y deseabilidad social (Eysenck, 1968). Riso (1988) y Riso, Pérez, Roldán y Ferrer (1988) reportan información sobre la adaptación y las propiedades psicométricas de la prueba para Colombia, donde su confiabilidad fue de 0.79 .

Escala de Estrategias de Coping Modificada (EEC-M)

Escala construida y estandarizada para Colombia por Londoño et al. (2006). El objetivo de esta prueba es identificar las estrategias de afrontamiento utilizadas por el evaluado; sus propiedades psicométricas han sido adecuadas hasta el momento, con un alfa de Cronbach de 0.85 .

\section{Prueba y cuestionarios nuevos}

Aunque las pruebas anteriormente descritas cubren la mayoría de las variables requeridas por la norma, las características sociodemográficas, ocupacionales, extralaborales y algunos riesgos psicosociales intralaborales no son medidos por los instrumentos descritos previamente; por esta razón se construyeron cuestionarios complementarios que evalúan las siguientes variables intralaborales: demandas físicas, exigencias de responsabilidad del cargo, demandas emocionales, demanda medioambiental, jornada de trabajo, participación y manejo del cambio, capacitación, claridad de rol y retroalimentación del desempeño.

Adicionalmente, se diseñaron preguntas con el fin de recabar la información sociodemográfica, extralaboral y ocupacional necesaria para contextualizar el resto de la información de cada trabajador. En la Tabla 4, más adelante, se resumen todos los factores evaluados, las pruebas con las cuales se miden y la estructura de la batería.

\section{Procedimiento}

Para desarrollar la batería se llevaron a cabo los siguientes pasos:

1. Se identificaron las variables de la Resolución 2646 de 2008 que podían ser valoradas mediante instrumentos de autorreporte.

2. Se buscaron instrumentos que midieran las variables identificadas y que cumplieran con los criterios establecidos previamente. En los casos en que no se encontraron instrumentos con los criterios definidos, se construyeron instrumentos nuevos. Para su construcción se realizó inicialmente una revisión de la literatura sobre cada variable y dos de los autores de este trabajo operacionalizaron las variables. Posteriormente se validó cada una de las definiciones con los demás autores. Una vez se alcanzó un consenso, se procedió con la redacción de los ítems. Cada ítem fue revisado y discutido por el grupo de investigadores hasta llegar a un acuerdo unánime.

3. Con la batería resultante, se realizó un estudio piloto con ocho trabajadores. Se evaluó el funcionamiento de la tecnología para la recolección de datos, la claridad de las instrucciones, las preguntas y opciones de respuesta.

4. Cada empresa participante envió a sus trabajadores un comunicado explicándoles el objeto de la investigación, las condiciones y formas de evaluación; a aquellos trabajadores que sabían leer, escribir y utilizar un computador se les envió un enlace a la batería para que contestaran las preguntas desde sus puestos de trabajo o en una sala de cómputo. Cuando el trabajador tenía dificultades de lectoescritura o con el uso de un computador, fue evaluado por un psicólogo utilizando la versión en papel de la batería. Un número muy pequeño de personas (83) necesitó este tipo de apoyo.

La primera parte del formulario consistía en el consentimiento informado; cuando se respondía afirmativamente a este, el sistema cargaba las preguntas de la batería o, en caso contrario, daba por finalizada la evaluación. A las personas que contestaron la prueba se les solicitó que registraran las sugerencias, dudas o dificultades que tuvieran durante su diligenciamiento, con el fin de obtener información cualitativa que permitiera análisis complementarios a los estadísticos. Finalmente, para realizar los análisis estadísticos se utilizó la versión 10 del SPSS. 


\section{RESULTADOS}

A continuación se presentan los resultados, divididos en dos etapas: la primera, que consistió en el análisis cualitativo, de confiabilidad y validez de constructo de los cuestionarios; y la segunda, en la que se llevaron a cabo los análisis de validez predictiva, concurrente y discriminativa, y el cálculo de las puntuaciones normalizadas.

Análisis cualitativo, de confiabilidad y validez de constructo

Los análisis cualitativos, de confiabilidad y validez de constructo se efectuaron para decidir qué ítems debían permanecer o no en la batería y para confirmar que los ítems se agrupaban de acuerdo con la estructura teórica de cada instrumento. La confiabilidad se estableció a partir del alfa de Cronbach y la validez de constructo se calculó mediante análisis factorial exploratorio con cada prueba.

Los criterios de inclusión de las escalas y los ítems fueron los siguientes: (a) que los comentarios no indicaran dificultades en la comprensión del ítem u opciones de respuesta, (b) que los alfas de Cronbach de la escala estuvieran entre 0.6 y 0.9 y (c) que el análisis factorial mostrara una estructura coherente con el modelo teórico. Se esperaba también que el peso factorial mínimo de cada ítem en su respectivo factor fuera de $0.3 \mathrm{y}$, en caso de pesar en dos factores diferentes, que ese peso fuera diferente en por lo menos 0.2. Adicionalmente, se analizaron los datos descriptivos (media, desviación estándar, mínimo y máximo) de cada ítem.

De los 2558 comentarios obtenidos, unos se referían a temas no relacionados con la prueba (quejas o comentarios particulares de cada empresa), otros a la extensión de la batería y a ítems de difícil comprensión (especialmente del inventario de personalidad) o repetitivos; estos ítems fueron analizados y se concluyó que en su mayoría estaban redactados de forma negativa, y la mayor parte hacían parte del inventario de personalidad.

Después de los análisis, se eliminaron 88 ítems de la batería, casi todos pertenecientes a las subescalas extroversión, psicoticismo y deseabilidad social del EPI y expresión de la dificultad de afrontamiento de la Escala de Coping Modificada. Con excepción de la subescala de neuroticismo, todas las demás del inventario de personalidad tuvieron bajos índices de confiabilidad. Algunos ítems del cuestionario nuevo para la evaluación complementaria de riesgo psicosocial se eliminaron porque eran repetitivos, y se concluyó que los ítems y las escalas restantes cumplían con los criterios de inclusión. En la Tabla 4 se encuentran los resultados descriptivos y de confiabilidad de todas las escalas que conforman la versión final de la batería.

Tabla 4.

Descriptivos y confiabilidad de las escalas

\begin{tabular}{|c|c|c|c|c|c|c|}
\hline Escala & Rango & Mín & Máx & Media & Desv. típ. & Alfa de Cronbach \\
\hline \multicolumn{7}{|l|}{ Factores psicosociales laborales } \\
\hline Indicadores de riesgo & & & & & & \\
\hline Tensión laboral a & 3 & 0 & 3 & 0.83 & 0.247 & $*$ \\
\hline Desequilibrio esfuerzo recompensa $^{b}$ & 3 & 0 & 4 & 0.91 & 0.288 & $*$ \\
\hline Inseguridad laboral a & 12 & 4 & 16 & 6.34 & 1.754 & 0.517 \\
\hline Demanda global $^{\mathrm{c}}$ & 169 & 52 & 221 & 121 & 19.482 & 0.889 \\
\hline Demandas psicológicas del trabajo $^{a}$ & 36 & 12 & 48 & 28.58 & 6.58 & 0.74 \\
\hline Demandas físicas ${ }^{\mathrm{c}}$ & 12 & 4 & 16 & 8.14 & 2.503 & 0.785 \\
\hline Esfuerzo extrínseco ${ }^{b}$ & 15 & 5 & 20 & 12.78 & 2.641 & 0.725 \\
\hline Exigencias de responsabilidad del cargo $^{c}$ & 15 & 5 & 20 & 13.86 & 2.673 & 0.765 \\
\hline Demandas emocionales $^{c}$ & 20 & 5 & 25 & 13.33 & 3.987 & 0.705 \\
\hline Demanda medioambiental $^{\mathrm{c}}$ & 24 & 6 & 30 & 11.32 & 3.83 & 0.652 \\
\hline Jornada de trabajo $^{\mathrm{c}}$ & 24 & 6 & 30 & 13.58 & 4.498 & 0.725 \\
\hline Control global ${ }^{\mathrm{c}}$ & 96 & 36 & 132 & 103.24 & 13.814 & 0.854 \\
\hline Participación y manejo del cambio ${ }^{c}$ & 16 & 4 & 20 & 14.63 & 3.73 & 0.872 \\
\hline Capacitación ${ }^{\mathrm{c}}$ & 8 & 2 & 10 & 7.82 & 2.001 & 0.886 \\
\hline Claridad de rol $^{\mathrm{c}}$ & 9 & 3 & 12 & 10.43 & 1.524 & 0.812 \\
\hline Control JCQ ${ }^{\mathrm{a}}$ & 72 & 24 & 96 & 71.15 & 10.681 & 0.777 \\
\hline Uso de habilidades ${ }^{\text {a }}$ & 36 & 12 & 48 & 37.82 & 5.317 & 0.701 \\
\hline Toma de decisiones ${ }^{\text {a }}$ & 36 & 12 & 48 & 33.33 & 6.953 & 0.693 \\
\hline Apoyo social global ${ }^{\mathrm{c}}$ & 36 & 11 & 47 & 36.53 & 5.767 & 0.874 \\
\hline Retroalimentación del desempeño $^{c}$ & 12 & 3 & 15 & 11.78 & 2.563 & 0.798 \\
\hline Apoyo social JCQ ${ }^{\text {a }}$ & 24 & 8 & 32 & 24.75 & 3.906 & 0.861 \\
\hline Apoyo del jefe o supervisor ${ }^{a}$ & 12 & 4 & 16 & 12.08 & 2.401 & 0.841 \\
\hline
\end{tabular}


(Cont. Tabla 4)

\begin{tabular}{|c|c|c|c|c|c|c|}
\hline Escala & Rango & Mín & Máx & Media & Desv. típ. & Alfa de Cronbach \\
\hline \multicolumn{7}{|c|}{ Factores psicosociales laborales } \\
\hline Apoyo de compañeros ${ }^{\text {a }}$ & 12 & 4 & 16 & 12.68 & 2.052 & 0.814 \\
\hline Recompensa $^{\mathrm{b}}$ & 33 & 11 & 44 & 31.91 & 4.872 & 0.823 \\
\hline \multicolumn{7}{|c|}{ Personalidad y afrontamiento } \\
\hline Esfuerzo intrínseco ${ }^{b}$ & 18 & 6 & 24 & 13.52 & 3.26 & 0.799 \\
\hline Neuroticismo $^{\mathrm{g}}$ & 16 & 0 & 16 & 4.26 & 3.297 & 0.81 \\
\hline Negación ${ }^{\mathrm{h}}$ & 15 & 3 & 18 & 8.38 & 2.897 & 0.605 \\
\hline Reacción agresiva $^{\mathrm{h}}$ & 25 & 5 & 30 & 8.86 & 3.345 & 0.75 \\
\hline Reevaluación positiva $^{\mathrm{h}}$ & 25 & 5 & 30 & 21.77 & 4.946 & 0.838 \\
\hline Búsqueda de apoyo social ${ }^{\text {h }}$ & 35 & 7 & 42 & 26.65 & 7.473 & 0.908 \\
\hline Búsqueda de apoyo profesional ${ }^{\mathrm{h}}$ & 25 & 5 & 30 & 12.75 & 6.114 & 0.905 \\
\hline Religión ${ }^{\mathrm{h}}$ & 35 & 7 & 42 & 26.75 & 8.486 & 0.876 \\
\hline Espera $^{\mathrm{h}}$ & 45 & 9 & 54 & 20.25 & 7.117 & 0.863 \\
\hline Solución de problemas ${ }^{\mathrm{h}}$ & 45 & 9 & 54 & 40.42 & 7.653 & 0.881 \\
\hline Evitación emocional ${ }^{\mathrm{h}}$ & 40 & 8 & 48 & 22.7 & 6.825 & 0.833 \\
\hline Evitación cognitiva $^{h}$ & 25 & 5 & 30 & 15.67 & 4.59 & 0.779 \\
\hline Autonomía ${ }^{\mathrm{h}}$ & 10 & 2 & 12 & 5.15 & 2.108 & 0.649 \\
\hline \multicolumn{7}{|c|}{ Salud, satisfacción y reacciones ante el estrés } \\
\hline Reacciones ante el estrés & 195 & 39 & 234 & 67 & 19.633 & 0.923 \\
\hline Salud general ${ }^{\mathrm{e}}$ & 20 & 5 & 25 & 19.99 & 3.448 & 0.837 \\
\hline Salud mental ${ }^{\mathrm{e}}$ & 25 & 5 & 30 & 25.34 & 4.298 & 0.836 \\
\hline Vitalidad $^{\mathrm{e}}$ & 20 & 4 & 24 & 18.21 & 3.855 & 0.866 \\
\hline Satisfacción laboral ${ }^{\mathrm{f}}$ & 16 & 4 & 20 & 15.1 & 2.833 & 0.806 \\
\hline
\end{tabular}

Nota: a. Cuestionario del contenido del trabajo JCQ. b. Cuestionario de desbalance esfuerzo recompensa DER. c. Cuestionario para la evaluación complementaria de riesgo psicosocial. d. Cuestionario para la medición de los factores de riesgos psicosociales. e. Cuestionario de Salud SF-36. f. Escala de satisfacción laboral de Whitehall. g. Inventario de personalidad EPI . h. Escala de Estrategias de Coping Modificada (EEC-M). * No hay cálculo de confiabilidad, ya que no es una única escala.

Cabe anotar que, aunque los niveles de confiabilidad de las variables inseguridad laboral y reacciones ante el estrés fueron de 0.52 y 0.93 respectivamente, por debajo o por encima de los valores ideales, se dejaron en la prueba final. En el caso de la inseguridad laboral se consideró que es una variable de alta relevancia en un contexto como el colombiano y que, además, no tuvo dificultades con otros indicadores psicométricos pues el valor alfa fue cercano al 0,6, el cual es aceptable (Aiken, 2003), y otros estudios han reportado valores similares (Araujo y Karasek, 2008; Cedillo y Karasek, 2003; Gómez, 2010; Gómez, 2011; Gómez y Perilla, 2011). En cuanto a las reacciones ante el estrés, aunque el valor alcanzado sugiere que la información ofrecida por los ítems fue redundante, esta situación era de esperarse en la medida en que el estrés se manifiesta en distintos sistemas orgánicos que es necesario explorar; el resultado es que la prueba contiene un gran número de ítems que se correlacionan altamente.

Los análisis factoriales de cada uno de los instrumentos confirmaron la estructura propuesta, con excepción del inventario de personalidad, pues de este solo la subescala de neuroticismo tuvo una estructura apropiada, con una buena confiabilidad, por lo que fue la única que quedó en la versión final de la batería.

\section{Análisis de validez y normalización}

Los análisis de validez predictiva, concurrente y discriminativa tuvieron como propósito determinar la capacidad de la batería para evaluar los aspectos para los cuales fue diseñada, relacionarse con otras variables de la manera teóricamente predicha y diferenciar grupos de acuerdo con lo esperado. Los baremos se calcularon para establecer rangos que permitieran comparar las puntuaciones de un individuo o grupo con aquellas obtenidas por otros trabajadores colombianos que se desempeñan en ocupaciones similares. Y, finalmente, se calcularon los rangos percentiles de cada variable de la batería.

Debido a que los análisis señalados previamente son sensibles a la composición de la muestra, era necesario asegurar que esta no estuviera sesgada y que fuera lo más representativa posible de las regiones y sectores económicos del país; por este motivo, se utilizó una submuestra de 2834 trabajadores seleccionados aleatoriamente y de forma proporcional al universo de trabajadores. La submuestra 
cubrió de forma adecuada la mayoría de cuotas requeridas por región y sector económico; todos los sectores y regiones estuvieron representados adecuadamente, excepto el sector agrícola.

\section{Validez predictiva}

Para evaluar la capacidad de los factores de riesgo psicosocial para relacionarse de la manera en que se predice teóricamente y como estudios previos han señalado que lo hacen, se calcularon correlaciones y regresiones lineales con los indicadores generales de las escalas JCQ y ERI, los factores de riesgo psicosociales globales (demanda, control y apoyo global) y la seguridad laboral como predictoras; y la salud general, la salud mental, la vitalidad, las reacciones ante el estrés y la satisfacción laboral como variables criterio. Los factores de riesgo psicosocial mostraron una tendencia de asociación positiva con las variables criterio, tal como se esperaba (véase Tabla 5). Sin embargo, la varianza explicada es mínima en algunos casos; por ejemplo, qué tanto de la salud general puede predecirse a partir del apoyo global. En otros casos, como el de la satisfacción laboral, casi el $40.96 \%$ de su varianza puede ser explicada a partir de las recompensas. Es interesante que las variables laborales que más varianza explican de varios indicadores de salud son el desequilibrio en los esfuerzos y las recompensas, y la inseguridad laboral. Por otro lado, la salud general es la variable en la que los factores laborales explican menor proporción de la varianza, lo cual no es sorprendente si se tiene en cuenta la multiplicidad de factores biomédicos y psicosociales que pueden determinarla.
En la tabla 6 se reportan los resultados de las regresiones que se calcularon con el fin de evaluar la capacidad de los indicadores de tensión laboral (TL) y desequilibrio esfuerzo-recompensa (DER) de predecir las variables de salud y bienestar. Además de los anteriores, se evaluó la capacidad de los indicadores globales (construidos con la nueva batería) y de la inseguridad laboral, de agregar explicación a la varianza más allá de TL y DER. Por otro lado, puesto que la edad ha demostrado, en estudio previos, tener una influencia directa sobre la salud general y sobre la satisfacción laboral, fue controlada en las regresiones calculadas con estos indicadores.

Los resultados muestran que la TL y el DER explican significativamente la varianza de todos indicadores de salud y bienestar seleccionados. El porcentaje de varianza que logran explicar es del $5 \%$ (TL) y el $2 \%$ (DER) para la salud general; del $10 \%$ y el $6 \%$ para las reacciones al estrés, la vitalidad y la salud mental; y del $16 \%$ y $13 \%$ en el caso de la satisfacción laboral. En todos los casos, la demanda global, el control global y la inseguridad laboral aumentaron significativamente la capacidad de predicción de las variables criterio, pero esta siempre fue inferior a la de TL y DER. Por otro lado, el apoyo social global ayudó significativamente a explicar, de manera directa, la salud mental, la salud general y la satisfacción general; y en el caso de las reacciones de estrés y la vitalidad, su aporte directo no fue significativo; sin embargo, es posible que en estos casos el papel del apoyo social sea más de moderador del impacto de las condiciones laborales, hipótesis que no se evaluó en este estudio.

$\underline{\text { Tabla } 5}$.

Correlaciones para el análisis de la validez predictiva

\begin{tabular}{|c|c|c|c|c|c|}
\hline $\begin{array}{l}\text { Indicadores de salud } \\
\text { Factores de riesgo }\end{array}$ & $\begin{array}{l}\text { Reacciones } \\
\text { ante el estrés }\end{array}$ & Salud general & Salud mental $^{+}$ & Vitalidad & Satisfacción laboral \\
\hline Tensión laboral ${ }^{\mathrm{a}+}$ & $0.12 * *$ & $-0.10^{* *}$ & $-0.09 * *$ & $-0.14 * *$ & $-0.13 * *$ \\
\hline Desequilibrio ${ }^{\mathrm{b}+}$ & $0.26 * *$ & $-0.14 * *$ & $-0.23 * *$ & $-0.24 * *$ & $-0.39 * *$ \\
\hline Inseguridad $^{+}$ & $0.24 * *$ & $-0.19 * *$ & $-0.23 * *$ & $-0.22 * *$ & $-0.32 * *$ \\
\hline Demanda global $^{\mathrm{c}}$ & $0.37 * *$ & $-0.17 * *$ & $-0.25 * *$ & $-0.26 * *$ & $-0.26^{* *}$ \\
\hline Control global ${ }^{\mathrm{d}}$ & $-0.11 * *$ & $0.09 * *$ & $0.08 * *$ & $0.14 * *$ & $0.31 * *$ \\
\hline Apoyo global ${ }^{\mathrm{e}}$ & $-0.03 * *$ & $0.06^{* *}$ & $0.09 * *$ & $0.06 * *$ & $0.16^{* *}$ \\
\hline Recompensas & $-0.37 * *$ & $0.27 * *$ & $0.34 * *$ & $0.36 * *$ & $0.64 * *$ \\
\hline
\end{tabular}

Nota: Algunas correlaciones se realizaron controlando variables de confusión. a Se controló el Desequilibrio Esfuerzo Recompensa. b Se controló la Tensión Laboral. c Se controlaron Control Global y Apoyo Global. d Se controlaron Apoyo Global y Demanda Global. e Se controlaron Demanda Global y Control Global. +La variable fue normalizada. ${ }^{* *} \mathrm{p}<0.01$ (bilateral). 


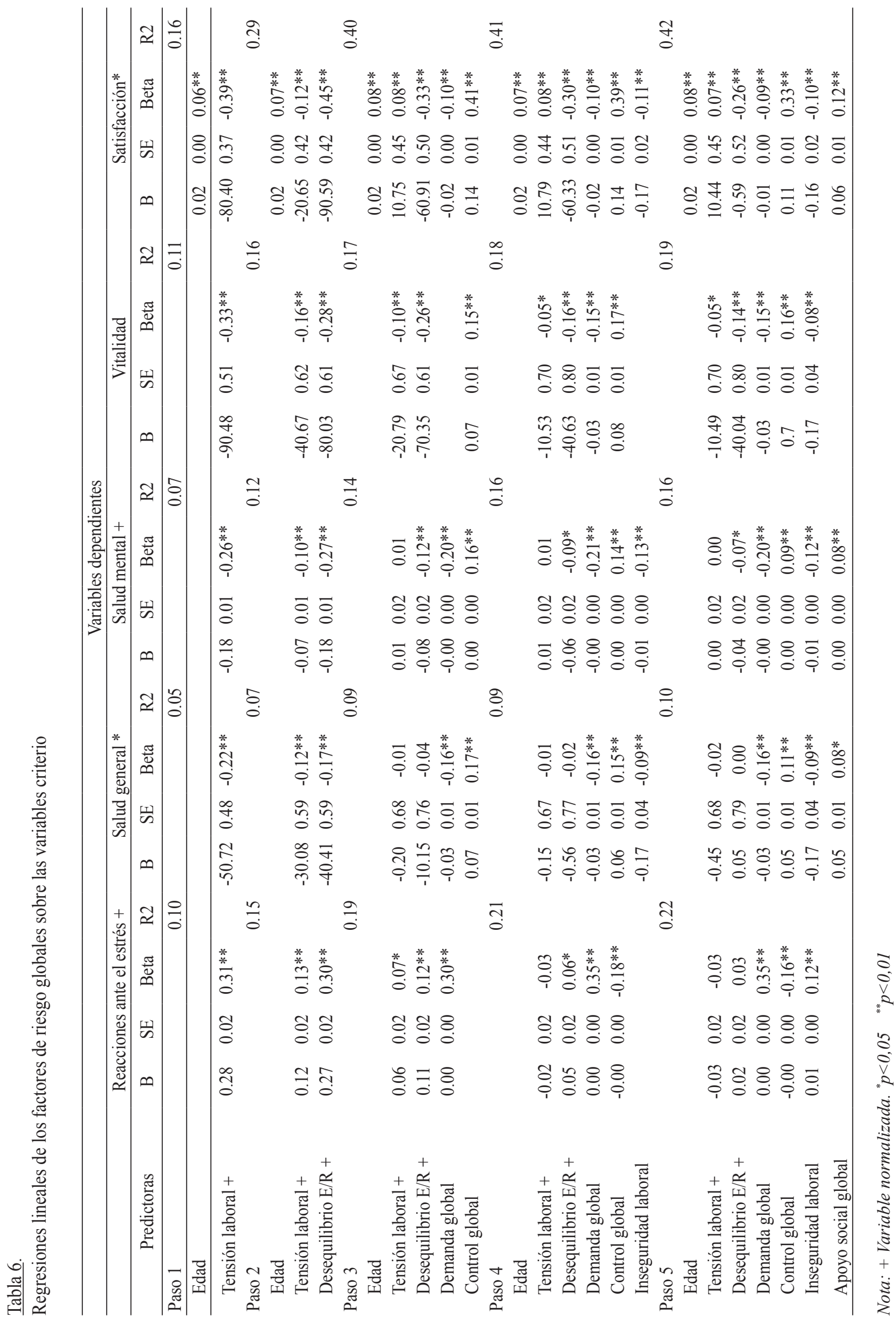




\section{Validez discriminativa}

Con este procedimiento se buscó establecer la capacidad del instrumento para diferenciar o discriminar trabajadores de grupos ocupacionales o de sectores económicos distintos, como teóricamente debería discriminar o como estudios previos han mostrado que lo hacen.

La validez discriminativa se calculó mediante análisis de varianza (ANOVA) utilizando los indicadores de TL, DER, demandas psicológicas, demandas físicas, demandas emocionales, control global, control JCQ, toma de decisiones, uso de habilidades, apoyo social global y apoyo social JCQ. En la Tabla 7 se observa cómo los grupos ocupacionales son discriminados por las variables psicosociales; por ejemplo, la TL diferencia trabajadores que desempeñan cargos profesionales, científicos e intelectuales de aquellos que desempeñan cargos operativos, estos últimos con más TL ( $\mathrm{p}<0.01)$; el control laboral medido con el JCQ y el control global diferencian los directivos de técnicos y operarios ( $\mathrm{p}<0.001$ ), estos últimos, como es de esperarse, reportan menos percepción de control; las demandas físicas discriminan los empleados en cargos directivos, técnicos, profesionales y científicos de los trabajadores con cargos operativos $(\mathrm{p}<0.01)$ cuyos niveles de demanda física son mayores; y, finalmente, las demandas emocionales son significativamente más altas en los profesionales que desempeñan cargos directivos y de servicios que en todos los demás trabajadores $(\mathrm{p}<0.01)$, como se esperaría teóricamente.
En cuanto al sector, se encontraron diferencias en TL, demandas físicas y emocionales, toma de decisiones y control (del JCQ). Los trabajadores del sector industrial reportan más TL que los del sector de servicios; los del sector comercio e industria se diferencian por mayores demandas físicas que los del sector servicios, tal como se esperaría; las demandas emocionales son mayores en el sector comercio y de servicios que los del sector industrial y agrícola, un resultado también esperable, y los trabajadores en el sector agrícola reportan menos control y toma de decisiones que en los demás sectores. Todas estas diferencias fueron significativas $(\mathrm{p}<0.01)$.

\section{Validez concurrente}

Con este procedimiento se buscó establecer si las variables que teóricamente miden conceptos cercanos, y que por tanto deben tener entre sí una correlación alta; se encontró que, efectivamente, tenían este tipo de relación. Los resultados de las correlaciones pueden apreciarse en las Tablas 8 y 9, que muestran niveles de asociación en la dirección y en los niveles esperados teóricamente. Por ejemplo, la tensión laboral y el desequilibrio esfuerzo-recompensa, dos conceptos cercanos, pero no idénticos, que reflejan aspectos psicosociales negativos del trabajo, correlacionan significativamente $(r=0.598)$; este valor es similar al obtenido por Gómez (2011) con muestras colombianas.

Tabla 7.

Análisis de discriminación por ocupación y por sector económico a partir de los factores de riesgo psicosociales evaluados

\begin{tabular}{lcccc}
\hline & \multicolumn{2}{c}{ Por ocupación } & \multicolumn{2}{c}{ Por sector } \\
& F (5.2828) & Sig. & F (3. 2830) & Sig. \\
\hline Tension Laboral + & 6.244 & 0.000 & 3.179 & 0.023 \\
Desequilibrio esfuerzo recompensa + & 5.921 & 0.000 & 0.685 & 0.561 \\
Control Global & 7.857 & 0.000 & 2.005 & 0.111 \\
Apoyo Social Global & 3.667 & 0.003 & 1.191 & 0.312 \\
Demandas físicas & 44.94 & 0.000 & 23.983 & 0.000 \\
Demandas emocionales & 14.197 & 0.000 & 14.423 & 0.000 \\
Uso de habilidades + & 21.822 & 0.000 & 1.578 & 0.193 \\
Demandas psicológicas del trabajo & 9.136 & 0.000 & 1.237 & 0.295 \\
Toma de decisiones & 23.574 & 0.000 & 11.938 & 0.000 \\
Control JCQ & 29.455 & 0.000 & 7.155 & 0.000 \\
Apoyo social JCQ & 2.156 & 0.056 & 1.301 & 0.272 \\
\hline
\end{tabular}

Nota: + Variable normalizada. 
Tabla 8.

Correlaciones para el análisis de la validez concurrente entre variables independientes.

\begin{tabular}{lcccc}
\hline & Tensión Laboral + & $\begin{array}{c}\text { Esfuerzo } \\
\text { extrínseco }\end{array}$ & $\begin{array}{c}\text { Jornada de } \\
\text { trabajo }\end{array}$ & $\begin{array}{c}\text { Retroalimentación del } \\
\text { desempeño }\end{array}$ \\
\hline $\begin{array}{l}\text { Desequilibrio esfuerzo recompensa }+ \\
\text { Demandas psicológicas del trabajo }\end{array}$ & $0.592^{* *}$ & & \\
Esfuerzo extrínseco & & $0.648^{* *}$ & $0.401^{* *}$ & \\
Apoyo del jefe o supervisor & & $0.458^{* *}$ & $0.571^{* *}$ \\
\hline
\end{tabular}

Variable normalizada $+{ }^{* *} \mathrm{p}<0.01$ (bilateral)

Tabla 9.

Correlaciones para el análisis de la validez concurrente entre variables dependientes

\begin{tabular}{lcccc}
\hline & $\begin{array}{c}\text { Reacciones ante el } \\
\text { estrés }+\end{array}$ & Salud mental + & Salud general & Vitalidad \\
\hline Reacciones ante el estrés + & $-0.624^{* *}$ & & \\
Salud mental + & $-0.468^{* *}$ & $0.409^{* *}$ & & \\
Salud general & $-0.632^{* *}$ & $0.670^{* *}$ & $0.513^{* *}$ & $0.452^{* *}$ \\
Vitalidad & $-0.386^{* *}$ & $0.369^{* *}$ & $0.308^{* *}$ & \\
Satisfacción laboral & & & \\
\hline
\end{tabular}

Variable normalizada $+* * \mathrm{p}<0.01$ (bilateral)

\section{Baremos}

Para ninguna de las variables que se contemplan en esta batería u otras similares existen puntos de corte establecidos empíricamente que permitan predecir, con toda precisión, qué personas efectivamente desarrollarán alguna enfermedad. Por lo tanto, la mejor forma disponible para establecer una valoración cualitativa de las puntuaciones es utilizar la distribución de la población como mecanismo de referencia para comparar los valores que obtenga una persona o grupo.

Para establecer los baremos se calcularon los valores mínimos, máximos, medios, desviaciones estándar y puntuaciones centiles de cada variable. Se utilizó la puntuación de percentiles, ya que permite, de forma muy rápida y fácil, establecer la posición relativa de cada evaluado o grupo respecto a un grupo de referencia. Los centiles se dividieron en 5 grupos para representar los niveles de frecuencia en la población: muy bajo, bajo, medio, alto y muy alto.

Cabe anotar que para el caso de los indicadores de riesgo TL y DER, otras investigaciones han señalado que las puntuaciones iguales o superiores a 1 se asocian con mayor riesgo y, en caso de mantenerse el nivel de exposición y conjugarse con condiciones de vulnerabilidad, con la aparición de enfermedades; por lo tanto, este es un punto de corte importante por considerar al margen de los resultados obtenidos en esta baremación (Gómez, 2010, 2011).

\section{DISCUSIÓN}

El objetivo de este trabajo fue construir y validar una batería que superara algunas de las limitaciones que se han identificado con la batería de factores de riesgo psicosocial del Ministerio de la Protección Social. Para cumplir con este propósito se diseñó una batería y se evaluaron su consistencia interna, su validez de constructo, predictiva, discriminatoria y concurrente. Adicionalmente, se calcularon puntuaciones normalizadas para Colombia que permitirán comparar los trabajadores evaluados con la población trabajadora del país, afiliada al sistema de riesgos laborales.

Los indicadores de calidad psicométrica (consistencia interna y validez de constructo) de todos los componentes de la batería son adecuados, resultado que coincide con estudios previos de instrumentos utilizados con muestras colombianas (Gómez, 2010, 2011; Londoño et al., 2006; Lugo et al., 2006; Riso, 1988; Riso et al., 1988) y confirma no solo que las escalas nuevas fueron diseñadas de manera apropiada, sino que la aplicación por computador no afecta la consistencia y validez de los instrumentos. Las 
escalas que evaluaron el neuroticismo (EPI), la satisfacción laboral (Whitehall II) y las reacciones ante el estrés (Cuestionario de factores de riesgo psicosocial) habían sido usadas en Colombia pero no se tenían datos publicados de su confiabilidad; en este estudio obtuvieron resultados que permiten respaldar su uso en el país.

Los indicadores de riesgo psicosocial incluidos en esta batería se asociaron de la forma esperada y significativa con los indicadores de percepción de salud general, salud mental, vitalidad, satisfacción laboral y con síntomas de estrés en una muestra de empleados representativa de los trabajadores afiliados al sistema de seguridad social. Cabe destacar los indicadores de TLy DER, los cuales explicaron la mayor proporción de la varianza de los indicadores de salud y bienestar utilizados. Al principio de este trabajo se argumentó que estos indicadores, a pesar de no tener en cuenta para su medición toda la información que la Resolución 2646 sugiere, permitirían predecir de manera económica riesgos para la salud tanto físicos como psicológicos. Efectivamente, los resultados dieron la razón a tal argumento inicial.

Los indicadores globales, construidos a partir de la información del JCQ y de las escalas nuevas, y la inseguridad laboral agregan explicación significativamente aunque la proporción no es tan alta (con excepción de la satisfacción laboral) de manera que los indicadores TL y DER podrían utilizarse, sin riesgo de estar perdiendo información que indique la necesidad de intervenir. Sin embargo, estos indicadores globales incluyen información adicional, sugerida por la Resolución 2646, que agregan detalle sobre las condiciones laborales que podrían ser objeto de atención preventiva y correctiva.

Adicionalmente, los indicadores laborales de riesgo discriminaron entre grupos ocupacionales y sectores económicos de la manera esperable, de acuerdo con el conocimiento que se posee sobre las características generales de estos grupos y sectores. El análisis de discriminación entre ocupaciones más específicas podrá ser desarrollado más adelante, a medida que se recojan suficientes datos de grupos ocupacionales específicos, algo que no puede hacerse con la batería existente. Estos análisis podrían compararse entonces con los que se han hecho en estudios previos (Karasek et. al., 1998).

Dados los resultados presentados en este documento, es posible afirmar que la batería que se construyó y evaluó supera las limitaciones de la batería del Ministerio que se enumeraron al principio de este trabajo: puede ser aplicada en papel y por computador; permitirá comparar ocupaciones; redujo la complejidad para la planeación y aplicación de las pruebas; permite obtener puntuaciones unificadas por variable; ofrece un diagnóstico de una gran cantidad de las variables sugeridas en la Resolución 2646, y permitirá comparar los resultados que se obtengan de grupos colombianos con los de trabajadores de otras partes del mundo, usando para ello los valores obtenidos en las escalas JCQ y ERI, incluidas de manera completa en esta batería.

La validación de esta batería tiene fortalezas destacables como el hecho de ser coherente teóricamente con los planteamientos de los modelos más sustentados en relación con su capacidad de predicción de problemas de salud de trabajadores de cualquier ocupación; o como que se hizo con una muestra de la población trabajadora afiliada al sistema de riesgos profesionales de un tamaño poco usual para este tipo de trabajos y que supera las limitaciones que puedan derivarse del hecho de haber sido escogida por conveniencia. Estos dos detalles agregan confianza y valor a la calidad de los resultados obtenidos y de las posibilidades que abren el uso de esta batería para las organizaciones que decidan utilizarla.

A pesar de las fortalezas señaladas, la batería tiene algunas limitaciones. Una de ellas se debe a que el sector agropecuario no está adecuadamente representado en la muestra, por lo cual, en caso de evaluar trabajadores de este sector, es necesario ser cuidadosos al compararlos con los representados en la batería. Otra limitación es el número de variables criterio que se utilizaron para su validación; en el futuro será necesario agregar otros indicadores de salud, en particular indicadores objetivos de salud mental y física, además de indicadores de desempeño. Adicionalmente, en la actualidad la batería es de acceso limitado puesto que los permisos otorgados por los autores de los cuestionarios JCQ y ERI no permiten, por ahora, la distribución o venta libre. Sin embargo, por el momento está siendo utilizada por una empresa privada (Empresa de Consultoría PRAX S.A.S) y por el grupo de investigación Estrés y Salud de la Universidad de los Andes (Bogotá, Colombia) para ofrecer servicios a las empresas interesadas y realizar trabajos de investigación que le permitan a la comunidad nacional tener información sobre algunas condiciones de salud y bienestar de la población afiliada al Sistema de Riesgos Laborales, además de su relación con condiciones psicosociales laborales. En el futuro se espera ampliar la información que se evalúa por medio de la batería, realizar investigaciones longitudinales y evaluar el impacto de las intervenciones. 


\section{REFERENCIAS}

Aiken, L. (2003). Tests psicológicos y evaluación. México: Prentice Hall.

Alvarado, R., Marchetti, N., Villalón, M., Hirmas, M., y Pastorino, M. S. (2009). Adaptación y análisis psicométrico de un cuestionario para evaluar riesgos psicosociales en el trabajo en Chile: versión media del CoPsoQ. Revista chilena de Salud Pública, 13(1), 7-16.

Araújo, T. y Karasek, R. (2008). Validity and reliability of the job content questionnaire in formal and informal jobs in Brazil. Scandinavian Journal of Work, Environment y Health, Supp. 6, 52-59.

Bahamón, N., y Zuluaga, L. (1993). Factores de riesgo psicosociales en los trabajadores de los institutos descentralizados afiliados a Capreneiva. Tesis de grado Especialización en Salud Ocupacional. Facultad de Salud Pública. Universidad de Antioquia.

Blanch, J. M., Sahagún, M., y Cervantes, G. (2010). Estructura Factorial del Cuestionario de Condiciones de Trabajo. Factor Structure of Working Conditions Questionnaire. Revista de Psicología del Trabajo y de las Organizaciones, 26(3), 175-189.

Bocanument, G. (1994). Naturaleza, origen, clasificación y evaluación de los riesgos psicosociales del trabajo. Administración de Personal, 16(52).

Bocanument, G., y Berjan, P. (1996). Encuesta para la identificación cuantitativa de los factores de riesgo psicosocial. En Seguro Social Protección Laboral, editor. Factores de riesgo psicosocial, 17-25. Documento técnico. Bogotá: ECOSAD.

Bosma, H., Peter, R., Siegrist, J., y Marmot, M. (1998). Two alternative job stress models and the risk of coronary heart disease. American Journal of Public Health January, 88(1), 68-74. Doi: 10.2105/AJPH.88.1.68

Brisson, C., Blanchette, C., Guimont, C., Dion, G., Moisan, J., y Vezina, M. (1998). Reliability and validity of the French version of the 18 item Karasek Job Content Questionnaire. Work and Stress, 12, 322-336.

Calnan, M., Wainwrite, D., y Almond, D. (2000). Job Strain, effortreward imbalance and mental distress: A study of occupations in general medical practice. Work y Stress, 14(4), 297-311.

Cedillo L., y Karasek R. (2003). Reliability and Validity of the Spanish Version of the Job Content Questionnaire Among Maquiladora Women Workers. Massachusetts: JCQ-Center. Recuperado de http://www.jcqcenter.org.

Comité Mixto OIT-OMS (1984). Factores Psicosociales en el Trabajo: Naturaleza, incidencia y prevención. Seguridad, Higiene y Medicina del Trabajo, 7.

Eum, K., Li, J., Lee, H., Kim, S., Paek, D., Siegrist, J., y Cho, S. (2007). Psychometric properties of the Korean version of the effort-reward imbalance questionnaire: a study in a petrochemical company. International Archives of Occupational and Environmental Health, 80(8), 653-661. Doi: 10.1007/ s00420-007-0174-3.
Eysenck, H. J. (1968). Eysenck personality inventory. San Diego: Educational and Industrial Testing Service.

Ganster, D. C., y Schaubroeck, J. (1991). Work stress and employee health. Journal of Management, 17, 235-271.

Gómez, V. (2010). Assessment of psychosocial stressor at work: Psychometric properties of the Spanish version of the ERI (Effort-Reward Imbalance Questionnaire) in Colombian Workers. Revista de Psicología del Trabajo y de las Organizaciones, 26(2), 147-156.

Gómez, V. (2011). Assessment of psychosocial stressors at work: psychometric properties of the JCQ in Colombian workers. Revista Latinoamericana de Psicología, 43(2), 125-138.

Gómez, V., y Perilla, E. (2011). Tensión laboral en varios grupos ocupacionales colombianos: Validación del modelo DemandaControl y del instrumento JCQ. Revista Ciencia y Trabajo, 13(42), 208-216.

Griep, R., Rotenberg, L., Vasconcellos, A., Landsbergis, P., Comaru, C., y Alves, M. (2009). The psychometric properties of demand-control and effort-reward imbalance scales among Brazilian nurses. International Archives of Occupational and Environmental Health, 82, 1163-1172. Doi: 10.1007/ s00420-009-0460-3.

Johnson, J.V., y Hall, E. (1988) Job strain, workplace social support and cardiovascular disease: A cross-sectional study of a random sample of the Swedish working population. American Journal of Public Health, 78(10), 1336-1342.

Johnson, J.V., Stewart, W., Hall E. M., Fredlund, P., y Theorell, T. (1996). Long-Term Psychosocial Work Environment and Cardiovascular Mortality among Swedish Men. American Journal of Public Health, 86(3), 325-331.

Johnson, J.V., Hall, E.M., y Theorell, T. (1989). Combined effects of job strain and social isolation on cardiovascular disease morbidity and mortality in a random sample of the Swedish male working population Scandinavian. Journal of Work, Environment \& Health, 15(4), 271-279.

Karasek R.A. (1979). Job demands, job decision latitude and mental strain: Implications for job redesign. Administrative Science Quarterly, 24, 285-307.

Karasek, R., Gardell, B., y Lindell, J. (1987). Work and nonwork correlates of illness and behaviour in male and female Swedish white collar workers. Journal of Organizational Behavior, 8, 187-207

Karasek, R., Brisson, Ch., Kawakami, N., Houtman, I., Bongers, P., y Amick, B. (1998). The Job Content Questionnaire (JCQ): An instrument for Internationally Comparative Assessments of Psychosocial Job Characteristics. Journal of Occupational Health Psychology, 3(4), 332-355.

Karasek, R., y Theorell, T. (1990). Healthy work. Stress, productivity and the reconstruction of Working Life. U.S.A: Basic Books. HarperCollins Pub.

Karasek, R.A., Baker, D., Marxer, F., Ahlbom, A., y Theorell, T. (1981). Job Decision Latitude, Job Demands, and Car- 
diovascular Disease: A Prospective Study of Swedish Men. American Journal of Public Health, 71(7), 694-705.

Kawakami, N., y Fujigaki, Y. (1996). Reliability and validity of the Japanese version Job Content Questionnaire: Replication and extension in computer company employees. Industrial Health, 34, 295-306.

Kawakami, N., Kobayahi, F., Araki, S., Haratani, T., y Furui , H. (1995). Assessment of job stress dimensions based on the job demands-control model of employees of telecommunication and electric power companies in Japan: Reliability and validity of the japanese version of the Job Content Questionnaire. International Journal of Behavioral Medicine, 4, 358-375.

Kinman, G., Jones, F., y Kinman, R. (2006). The Well-being of the UK Academy, 1998-2004. Quality in Higher Education, 12(1), 15-27.

Kristensen, T. S. (1995). The Demand-Control-support model: Methodological challenges for future research. Stress Medicine, 11, 17-26.

Kristensen, T. S. (1996). Job stress and cardiovascular disease: A theoretic critical review. Journal of Occupational Health Psychology, 1(3), 246-60.

Lazarus, R. (1999). Stress and Emotion: A New Synthesis. Nueva York: Springer Publishing Company.

Londoño, N. H., Henao López, G. C., Puerta, I. C., Posada, S., Arango, D., y Aguirre-Acevedo, D. C. (2006). Propiedades psicométricas y validación de la Escala de Estrategias de Coping Modificada (EEC-M) en una muestra colombiana. Universitas Psychologica, 5(2), 327-350.

Lugo, L. H., García, H. I., y Gómez, C. (2006). Confiabilidad del cuestionario de calidad de vida en salud SF-36 en Medellín, Colombia. Rev. Fac. Nac. Salud Pública, 24(2), 37-50.

Lundberg, U., y Cooper, C. L. (2011). The science of occupational health. Chichester, UK: Wiley-Blackwell.

Macías Robles, M. D., Fernández López, J. A., Hernández Mejía, R., Cueto Espinar, A., Rancaño, I., y Siegrist, J. (2003). Evaluación del estrés laboral en trabajadores de un hospital público español. Estudio de las propiedades psicométricas de la versión española del modelo "Desequilibrio esfuerzorecompensa". Medicina Clínica, 120(17), 652-657.

Marmot, M. (2013). Whitehall II History. UCL Research Department of Epidemiology and Public Health Web Site. Recuperado de http://www.ucl.ac.uk/whitehallII/history

Ministerio de la Protección Social (2008). Resolución 2646 de julio 17 de 2008. Diario Oficial 47059 de julio 23.

Ministerio de la Protección Social, Pontificia Universidad Javeriana (2010). Batería de instrumentos para evaluación de factores de riesgo psicosocial. Manual general de la batería de instrumentos. Bogotá: Ministerio de la Protección Social República de Colombia. Recuperado de http://fondoriesgoslaborales.gov.co/documents/Publicaciones/Estudios/ Bateria-riesgo-psicosocial-1.pdf

Ministerio de la Protección Social. (2011). Respuesta a derecho de petición número 00279985 del 15 de septiembre de 2011 .
Moncada, S., Llorens, C., y Kristensen, T. S. (2004). Método ISTAS 21 (CoPsoQ). Manual para la evaluación de riesgos psicosociales en el trabajo. Instituto Sindical de Trabajo, Ambiente y Salud (ISTAS). Paralelo Ediciones SA.

Msaouel, P., Keramaris, N., Apostolopoulos, A., Syrmos, N., Kappos, T., Tasoulis, A., Tripodaki, E.-S., Kagiampaki, E., Lekkas, I., y Siegrist, J. (2012). The Effort-reward Imbalance Questionnaire in Greek: Translation, Validation and Psychometric Properties in Health Professionals. Journal of Occupational Health, 54 (2), 119-130.

Niedhammer, I., Siegrist, J., Landre, M., Goldberg, M., y Leclerc, A. (2000). Etude des qualités psychométriques de la version francaise du modèle d'équilibre Efforts/Recompenses. Revue d'Epidemiologie et de Santé Publique, 48, 419-437.

Nübling, M., Stößel, U., Hasselhorn, H. M., Michaelis, M., y Hofmann, F. (2006). Measuring psychological stress and strain at work-evaluation of the COPSOQ Questionnaire in Germany. GMS Psycho-Social Medicine, 3, 1-14.

Pejtersen, J. H., Kristensen, T. S., Borg, V., y Bjorner, J. B. (2010). The second version of the Copenhagen Psychosocial Questionnaire. Scandinavian Journal of Public Health, $38(3$ supp.), 8-24.

Pelfrene, E., Vlerick, P., Mak, R., De Smet, P., Kornitzer, M., y De Backer, G. (2001). Scale reliability and validity of the Karasek' Job Demand-Control-Support model in the Belstress study. Work and Stress, 4, 297-313.

Peter, R., Siegrist, J. Hallqvist, J., Reuterwall, C., y Theorell, T. (2002). Psychosocial work environment and myocardial infarction: Improving risk estimation by combining two complementary job stress models in the SHEEP Study. Journal of Epidemiology and Community Health, 56, 294-300.

Riso, W. (1988). Entrenamiento asertivo: aspectos conceptuales, evaluativos y de intervención. Medellín: Formar.

Riso, W., Pérez, G. M., Roldán, L., y Ferrer, A. (1988). Diferencias en ansiedad social, creencias irracionales y variables de personalidad en sujetos altos y bajos en asertividad (tanto en oposición como en afecto). Revista Latinoamericana de Psicología, 20(3), 391-400.

RLDatos-FASECOLDA-(2013). Reporte de Trabajadores afiliados al sistema de riesgos laborales. Recuperado de https://sistemas.fasecolda.com/rldatos/Reportes/xClaseGrupoActividad.aspx

Sale, J. E. M., y Kerr, M. S. (2002). The psychometric properties of Karasek's demand and control scales within a single sector: data from a large teaching hospital. International Archives of Occupational Environmental Health, 75, 145-152.

Sarsosa, K. V., Arenas, F., y Charria, V. H. (2011). Factores de riesgo psicosocial laboral: métodos e instrumentos de evaluación. Revista Facultad Nacional de Salud Pública, 29(4), 380-391.

Semmer, N. K., y Meier, L. L. (2009). Individual differences, work stress, and health. En C. L. Cooper, J. Campbell Quick, y M. J. Schabracq (eds.), International handbook of work and health psychology ( $3^{\text {rd }}$ ed., pp. 99-121). Chichester, UK: Wiley. 
Siegrist, J. (2002). Effort-Reward Imbalance at Work and Health. En: P.L. Perrewé y D.C. Ganster(Eds.). Historical and Current Perspectives on Stress and Health, vol. 2, 261-291. Emerald Group Publishing Limited.

Schnall, P. L., Belkic, K., Landsbergis, P. L., y Baker, D. (2000). Why the workplace and cardiovascular disease? In P. L. Schnall, K. Belkic, P. Landsbergis, y D. Baker (Eds.). Occupational Medicine: State of the Art Reviews, 15(1), Filadelfia: Hanley and Belfus.

Sverke, M., y Hellgren, J. (2002). The Nature of Job Insecurity: Understanding Employment Uncertainty on the Brink of a New Millennium. Applied Psychology: An International Review, 51(1), 23-42.

Sverke, M., Hellgren, J., y Näswall, K. (2002). No security: A metaanalysis and review of job insecurity and its consequences. Journal of Occupational Health Psychology, 7(3), 242-264.

Theorell, T., y Karasek, R. A. (1996). Current issues relating to psychosocial job strain and cardiovascular disease research. Journal of Occupational Health Psychology, 1, 9-26.

Tsutsumi, A., Kayaba, K., Theorell, T., y Siegrist, J. (2001). Association between job stress and depression among Japanese employees threatened by job loss in a comparison between two complementary job-stress models. Scandinavian Journal of Work, Environment y Health, 27(2), 146-153.

Turner-Bowker, D.M., Bartley, P.J., y Ware, J.E., Jr. (2002). SF-36® Health Survey y “SF” Bibliography: Third Edition (1988-2000). Lincoln, RI: QualityMetric Incorporated.

Van der Doef, M., y Maes, S. (1999). The Job Demand-Control (-Support) Model and psychological well-being: a review of 20 years of empirical research. Work Stress, 13, 87-114.

Villalobos, G. H., Vargas, A. M., Rondón, M. A., y Felknor, S. A. (2013). Design of psychosocial factors questionnaires: A systematic measurement approach. American Journal of Industrial Medicine, 56(1), 100-110.

Ware, J. E. (2013). SF-36® Health Survey Update. The SF Community. Recuperado de http://www.sf-36.org/tools/sf36.shtml

Winefield, A., Gillespie, N., Stough, C., Dua, J., Hapuarachchi, J., y Boyd, C. (2003). Occupational stress in australian university staff: Results from a national survey. International Journal of Stress Management, 10 (1), 51-63. 